Chinese Journal of Organic Chemistry

NOTE

\title{
钯催化合成氧化芪三酚的研究
}

\author{
李志伟*, $a$ 康绍英 ${ }^{b}$ 陈 琳 ${ }^{a}$ 王 宇 ${ }^{a}$ 李江胜 $*, a$ \\ $\left({ }^{a}\right.$ 电力与交通材料保护湖南省重点实验室 长沙理工大学化学与生物工程学院 长沙 410114)

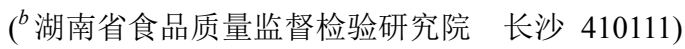

\begin{abstract}
摘要 研究了一种钯催化合成氧化花三酚的方法. 2,4-二羟基碘苯与丙烯酸在钯催化剂的作用下发生 Heck 反应和脱羧 反应, 再与 3,5-二羟基碘苯发生 Heck 反应, 以较高产率得氧化花三酚. 目标产物的结构经 MS, ${ }^{1} \mathrm{H} \mathrm{NMR}$ 及 ${ }^{13} \mathrm{C} N \mathrm{NMR}$ 确 证. 该方法具有合成步骤少、产率高和操作简单等特点, 有望实现工业化生产.

关键词 氧化花三酚; 钯催化; Heck 反应; 脱羧反应
\end{abstract}

\section{Study on the Synthesis of Oxyresveratrol Catalyzed by Palladium}

\author{
Li, Zhiwei*a ${ }^{*, a}$ Kang, Shaoying ${ }^{b} \quad{\text { Chen, } \text { Lin }^{a} \quad \text { Wang, Yu }}^{a} \quad$ Li, Jiangsheng ${ }^{*, a}$ \\ ( ${ }^{a}$ Hunan Provincial Key Laboratory of Materials Protection for Electric Power and Transportation, School of Chemistry and \\ Biological Engineering, Changsha University of Science and Technology, Changsha 410114) \\ ( ${ }^{b}$ Hunan Institute of Food Quality Supervision Inspection and Research, Changsha 410111)
}

\begin{abstract}
A convenient method for the synthesis of oxyresveratrol catalyzed by palladium is described. 2,4-Dihydroxyiodobenzene underwent Heck reaction with acrylic acid, then decarboxylation, and further underwent Heck reaction with 3,5-dihydroxyiodobenzene, furnishing oxyresveratrol in a high yield. The title compound was characterized by MS and NMR. This synthetic method has several advantages such as less step processes, high yield, simple operations, and promises to be applied in industrial production.
\end{abstract}

Keywords oxyresveratrol; palladium catalysis; heck reaction; decarboxylation

氧化芪三酚(又叫氧化白藜芦醇，英文名：Oxyresveratrol, $E-2,3^{\prime}, 4,5^{\prime}$-tetra-hydroxystilbene)是多羟基反 式苯乙烯类天然产物芪三酚的 2'-位羟基化派生物. 已 有研究表明，氧化芪三酚具有高效的抑制酪氨酸酶活性 和皮肤色素沉淀作用, 能抵抗疮疹、防御病毒、减轻氧 化、守护神经以及发生脑缺血时减少细胞凋亡的作用. 加上该化合物具有低毒性和较高的水溶性, 因此它有着 优良的药物开发潜力, 如美白抗氧化、抗炎、抗血栓、 抗癌、抗高血脂症和抗菌等. 随着研究开发的不断深入, 氧化芪三酚有望在保健品、医学以及果蔬保鲜等领域获 得广泛应用 ${ }^{[1-3]}$. 在自然界中, 氧化芪三酚主要存在于 桑科中的波罗蜜属、桑属、橙桑属、菝荰属、愁芦属及 买麻藤属等珍稀植物中 ${ }^{[4,5]}$, 含量非常低, 提取困难, 从 而导致其天然来源十分有限. 因此, 用化学合成的方法
来获取氧化芪三酚是解决其来源的有效途径.

目前合成氧化芪三酚的方法鲜有报道. 2004 年, Kim 等 ${ }^{[6]}$ 运用 3,5-二甲氧基苄基溴与三苯基膦反应后, 再与 2,4-二甲氧基苯甲醛发生 Witing 反应, 然后在碘的 作用下生成反式烯烃, 最后在格氏试剂的作用下脱去甲 基得目标产物. 2010 年, 邹永等 ${ }^{[7]}$ 以 3,5-二羟基苯乙酮 为原料，经甲基化、Willgerodt-Kindler 重排以及 Perkin 反应构建二苯乙烯骨架, 再经脱羧及脱甲基异构化得目 标产物. 2013 年, 郑群怡等 ${ }^{[8]}$ 运用 3,5-二甲氧基芐溴与 亚磷酸三乙酯反应后，再与 2,4-二甲氧基苯甲醛发生 Witting-Horner 反应得 $E-2,3^{\prime}, 4,5^{\prime}$-四甲氧基二苯乙烯. 最 后在格氏试剂的作用下脱去甲基得目标产物. 但是这几 种方法存在合成路线比较长、总收率比较低和操作危险 性大等缺点. 因此, 研究一种简单和高效地制备氧化花

*E-mail:lizwhn@126.com; jslichem@gmail.com

Received September 30, 2015; revised December 3, 2015; published online January 4, 2016.

Project supported by the Natural Science Foundation of Hunan Province (No. 2015JJ3012), the Scientific Research Fund of Hunan Provincial Education Department (No. 14C0050) and Hunan Provincial Key Laboratory of Materials Protection for Electric Power and Transportation (2016CL01). 湖南省自然科学基金(No. 2015JJ3012)、湖南省教育厅(No. 14C0050)和电力与交通材料保护湖南省重点实验室(No. 2016CL01)资助项目. 
三酚的方法具有非常重要的意义.

钯催化的 Heck 反应 ${ }^{[9,10]}$ 和脱羧反应 ${ }^{[11,12]}$ 在有机合成 中得到了广泛的应用. 由于烯烃存在难以合成、纯化困 难且容易聚合等问题 ${ }^{[13]}$, 所以原位合成烯烃并运用于 Heck 反应的方法也得到了广泛的研究和应用 ${ }^{[14]}$.

本文从 3,5-二甲氧基苯胺出发, 经过碘代反应和脱 甲基反应后得 3,5 -二羟基碘苯(1). 从间苯二酚出发, 经 过碘化反应后得 2,4-二羟基碘苯(2), 然后与丙烯酸在钯 催化剂的作用下发生 Heck 反应和脱羧反应, 得到原位 生成的中间体 2,4-二羟基苯乙烯(3). 3 在钯催化下与 3,5二羟基碘苯(1)发生第二次 Heck 反应, 得到目标产物氧 化芪三酚. 合成路线如 Scheme 1 所示.<smiles>COc1cc(N)cc(OC)c1</smiles>
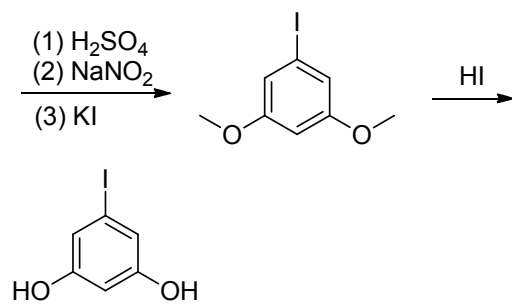

1
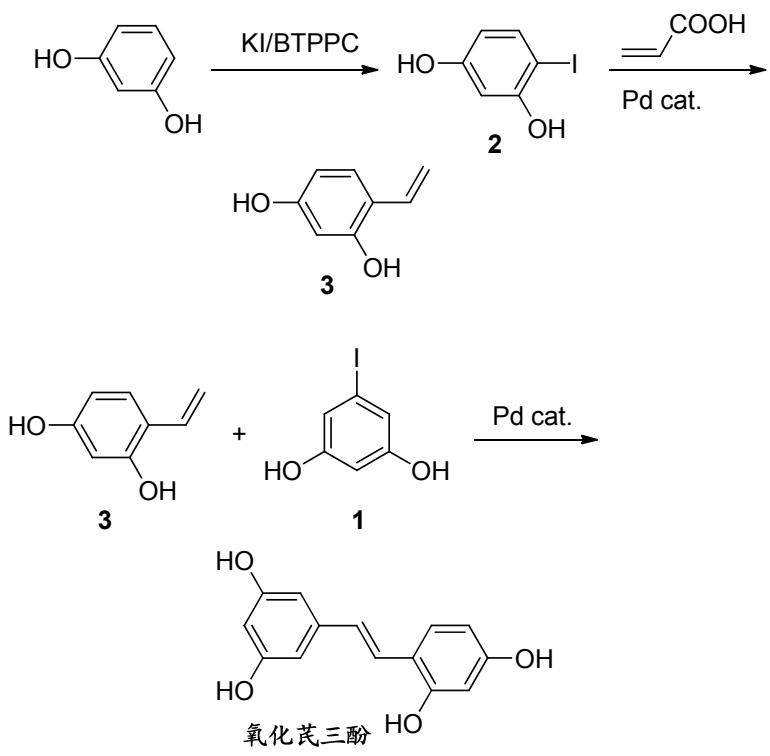

图式 1 目标化合物的合成路线

Scheme 1 The synthetic route of target compound

\section{1 结果与讨论}

\section{1 合成讨论}

在目标产物路线设计时, 我们考虑到, 如果三组分 (3,5-二羟基碘苯、 2,4 -二羟基碘苯和丙烯酸)在钯催化剂 存在下采用一锅煮的方法, 3,5-二羟基碘苯将与丙烯酸 反应, 生成的 3,5-二羟基苯乙烯再与未反应的 3,5-二羟 基碘苯反应而生成副产物. 因此, 我们设计分步反应的
方法: 先用 2,4-二羟基碘苯与丙烯酸在钯催化剂的作用 下发生 Heck 反应和脱羧反应，原位生成中间体 2,4-二 羟基苯乙烯后, 再加入 3,5-二羟基碘苯发生第二次 Heck 反应得目标产物.

首先以醋酸钯为催化剂, 氢氧化钾为碱, $N, N$-二甲 基甲酰胺为溶剂, 2,4-二羟基碘苯与丙烯酸在 $120{ }^{\circ} \mathrm{C}$ 下 反应 $12 \mathrm{~h}$ 后, 发现反应很慢. 通过改变溶剂体系, 发现 该反应在水中回流反应 $3 \mathrm{~h}$ 就可完成. 降低反应温度到 $85{ }^{\circ} \mathrm{C}$, 反应也能较好地进行; 继续降低反应温度至 $60{ }^{\circ} \mathrm{C}$, 反应进行得很慢. 因此, 我们选择 2,4-二羟基碘 苯与丙烯酸在 $85{ }^{\circ} \mathrm{C}$ 下反应 $3 \mathrm{~h}$ 后再往反应体系中加入 3,5-二羟基碘苯, 并继续在 $85{ }^{\circ} \mathrm{C}$ 下反应, 发现反应进行 得很慢. 提高反应温度至 $100{ }^{\circ} \mathrm{C}$, 效果仍然不理想. 当 往体系中加入 $N, N$-二甲基甲酰胺后发现反应能较好地 进行. 通过优化 $N, N$-二甲基甲酰胺的用量以及反应温 度, 发现在 4 equiv. 的 $N, N$-二甲基甲酰胺和 $140{ }^{\circ} \mathrm{C}$ 下反 应能较好地进行, 目标产物收率为 $22.3 \%$.

研究了碱、溶剂、钯催化剂以及添加剂等对合成目 标产物的影响. 首先研究了碳酸钾、三乙胺和哌啶等碱 对反应的影响. 发现该反应在碳酸钾和三乙胺的作用 下, 产率有较大的下降(表 1, Entries 2,3); 而在哌啶的 作用下，产率有较大的提高(表 1, Entry 4). 以哌啶为碱, 研究了 $N, N$-二甲基乙酰胺和 $N$-甲基吡咯烷酮等其它溶 剂对反应的影响. 发现该反应在 $N, N$-二甲基乙酰胺中, 产率有较大的提高(表 1, Entry 5). 紧接着研究了不同钯 催化剂如氯化钯、三氟乙酸钯以及四 (三苯基膦) 钯等 对反应的影响. 发现该反应在氯化钯和三氟乙酸钯的催 化下产率均有较大的下降(表 1, Entries 7,8), 而在四 (三 苯基膦）钯的催化下产率有较大的提高(表 1, Entry 9). 还研究了添加剂对反应的影响, 发现氯化锂的加入能使 产率有小幅的提高(表 1, Entry 10), 而碳酸铯的加入使 产率有小幅的下降(表 1, Entry 11). 研究结果如表 1 所 示.

以四(三苯基膦)钯为催化剂、哌啶为碱、氯化锂为 添加剂和 $N, N$-二甲基乙酰胺为溶剂, 研究了氧气、催化 剂用量、反应温度和反应时间对产率的影响. 结果表明, 该反应在氮气下能更好地进行, 产率有所提高, 达到 $60.5 \%$ (表 2, Entry 2), 但是操作非常繁琐. 在氮气氛围 中, 将钯催化剂的用量降低到 $3.5 \%$, 产率只有少量的降 低(表 2, Entry 3). 但是在空气中将钯催化剂的用量降低 到 3.5\%, 产率降低显著(表 2, Entry 4). 缩短反应时间和 降低反应温度, 产率均出现较大的下降(表 2, Entries 5 , 6). 另外, 还考察了该反应放大后的效果, 发现反应规 模放大 10 倍以后，产率还有少量提高(表 2, Entry 7)。该 反应有望实现工业化生产. 结果如表 2 所示. 
表 1 笁选不同的碱、溶剂、钯催化剂以及添加剂 ${ }^{a}$

Table 1 Screening of different bases, solvents, palladium catalysts and additives

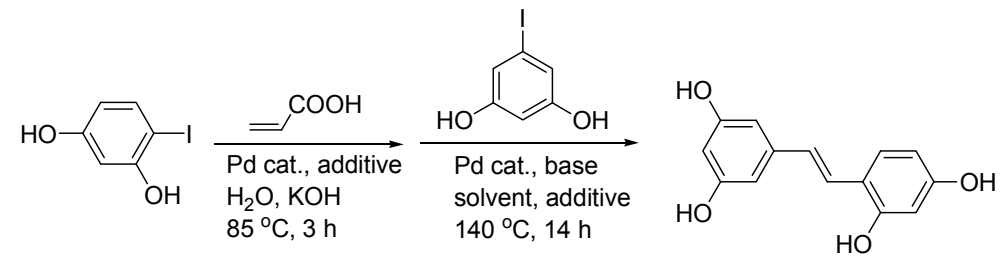

\begin{tabular}{|c|c|c|c|c|c|}
\hline Entry & Base & Solvent & Pd catalyst & Additive & Yield $/ \%$ \\
\hline 1 & $\mathrm{KOH}$ & DMF & $\mathrm{Pd}(\mathrm{OAc})_{2}$ & - & 22.3 \\
\hline 2 & $\mathrm{~K}_{2} \mathrm{CO}_{3}$ & DMF & $\mathrm{Pd}(\mathrm{OAc})_{2}$ & - & 10.5 \\
\hline 3 & $\mathrm{Et}_{3} \mathrm{~N}$ & DMF & $\mathrm{Pd}(\mathrm{OAc})_{2}$ & - & 12.4 \\
\hline 4 & Piperidine & DMF & $\mathrm{Pd}(\mathrm{OAc})_{2}$ & - & 31.2 \\
\hline 5 & Piperidine & DMA & $\mathrm{Pd}(\mathrm{OAc})_{2}$ & - & 38.6 \\
\hline 6 & Piperidine & NMP & $\mathrm{Pd}(\mathrm{OAc})_{2}$ & - & 24.5 \\
\hline 7 & Piperidine & DMA & $\mathrm{PdCl}_{2}$ & - & 22.4 \\
\hline 8 & Piperidine & DMA & $\mathrm{Pd}\left(\mathrm{CF}_{3} \mathrm{COO}\right)_{2}$ & - & 25.6 \\
\hline 9 & Piperidine & DMA & $\mathrm{Pd}\left(\mathrm{PPh}_{3}\right)_{4}$ & - & 54.5 \\
\hline $10^{c}$ & Piperidine & DMA & $\mathrm{Pd}\left(\mathrm{PPh}_{3}\right)_{4}$ & $\mathrm{LiCl}$ & 57.1 \\
\hline $11^{c}$ & Piperidine & DMA & $\mathrm{Pd}\left(\mathrm{PPh}_{3}\right)_{4}$ & $\mathrm{Cs}_{2} \mathrm{CO}_{3}$ & 50.3 \\
\hline
\end{tabular}

${ }^{a}$ Unless stated otherwise, all reactions were performed as follows: acrylic acid $(2.0 \mathrm{mmol})$, 4-iodobenzene-1,3-diol (1.8 mmol), palladium catalysts (4.0 mol\%), KOH $(6.0 \mathrm{mmol})$ and water $(2.0 \mathrm{~mL})$ were stirred at $85{ }^{\circ} \mathrm{C}$ for $3 \mathrm{~h}$, then 5-iodobenzene-1,3-diol $(1.8 \mathrm{mmol})$, base $(4.0 \mathrm{mmol})$, palladium catalyst $(3.0 \mathrm{~mol} \%)$, solvent $(8.0$ $\mathrm{mL}$ ) were added and stirred at $140{ }^{\circ} \mathrm{C}$ for further $14 \mathrm{~h} ;{ }^{b}$ Isolated yield; ${ }^{c} 8 \mathrm{~mol} \%$ additive was added at the first step and $3 \mathrm{~mol} \%$ at the second step.

\section{表 2 反应条件对产率的影响 ${ }^{a}$}

Table 2 Effect of reaction conditions on the yield of the product

\begin{tabular}{cccccc}
\hline Entry & Air/ $\mathrm{N}_{2}$ & Cat. loading $/ \%$ & Temp. $/{ }^{\circ} \mathrm{C}$ & $t / \mathrm{h}$ & $\mathrm{Yield}^{b} / \%$ \\
\hline 1 & Air & 7.0 & 140 & 14 & 57.1 \\
2 & $\mathrm{~N}_{2}$ & 7.0 & 140 & 14 & 60.5 \\
3 & $\mathrm{~N}_{2}$ & 3.5 & 140 & 14 & 56.3 \\
4 & Air & 3.5 & 140 & 14 & 40.8 \\
5 & Air & 7.0 & 140 & 8 & 48.3 \\
6 & Air & 7.0 & 100 & 14 & 42.3 \\
$7^{c}$ & Air & 7.0 & 140 & 14 & 59.2 \\
\hline
\end{tabular}

${ }^{a}$ Unless stated otherwise, all reactions were performed as follows: acrylic acid (4.16 mmol), 4-iodobenzene-1,3-diol (3.74 mmol), $\mathrm{Pd}\left(\mathrm{PPh}_{3}\right)_{4}$ (4.0 mol\%), $\mathrm{LiCl}$ $(8.0 \mathrm{~mol} \%), \mathrm{KOH}(12.48 \mathrm{mmol})$ and water $(3 \mathrm{~mL})$ were stirred at $85{ }^{\circ} \mathrm{C}$ for 3 $\mathrm{h}$, then 5-iodobenzene-1,3-diol $(3.74 \mathrm{mmol})$, piperidine $(8.32 \mathrm{mmol})$, $\mathrm{Pd}\left(\mathrm{PPh}_{3}\right)_{4}(3.0 \mathrm{~mol} \%), \mathrm{LiCl}(3.0 \mathrm{~mol} \%)$, DMA $(12.0 \mathrm{~mL})$ were added and stirred at $140{ }^{\circ} \mathrm{C}$ for further $14 \mathrm{~h} ;{ }^{b}$ Isolated yield; ${ }^{c} 10$ equiv. of the starting material.

\section{2 目标化合物的图谱解析}

分析目标产物的 ${ }^{1} \mathrm{H}$ NMR 谱图可以得知, 在 $\delta 6.06$, $6.23,6.31,6.33,6.76,7.14,7.34,9.17,9.41,9.60$ 共有 10 组峰 12 个氢, 其中 $\delta 9.17,9.41,9.60$ 的三组峰共四个氢 归属为羟基上的氢; $\delta 6.76$ 和 7.14 的两组峰归属为烯烃 上的两个氢, 且这两组峰为典型的 $\mathrm{AB}$ 体系 $\mathrm{dd}$ 峰, 经过 计算这两个氢之间的偶合常数为 16.4 , 是典型的碳碳双 键反式烯烃上的氢. ${ }^{1} \mathrm{H}$ NMR 谱中的峰的种类、个数和峰 形与氧化芪三酚的结构以及文献[7]报道相符. 分析目 标产物的 ${ }^{13} \mathrm{C} \mathrm{NMR}$ 谱图可以得知, 在 $\delta 101.5,102.8$, $104.7,107.6,116.4,123.5,125.5,127.4,140.8,156.0$, 158.2, 158.6 共 12 种碳, 与氧化芪三酚的结构相符. 分
析目标产物 ESI 质谱图可以得知，该化合物的分子量 是 244 , 与氧化芪三酚的分子量相符. 由此可以判断, 得到的是目标产物氧化芪三酚.

\section{3 合成目标产物可能的反应机理}

我们推测合成目标产物的反应机理如图 1 所示. 2,4-二羟基碘苯在钯催化下与丙烯酸发生第一次 Heck 反应, 生成肉桂酸衍生物中间体 I1, I1 可能在碱的作用 下转换为醌式结构的中间体 $\mathbf{I}, \mathbf{I} 2$ 发生脱羧反应得到中 间体 1. I1 也有可能在钯催化剂的作用下直接发生脱羧 反应生成中间体 $\mathbf{1}^{[11,12]}$. 3,5-二羟基碘苯在钯催化剂的作 用下生成中间体 I3, I3 与中间体 1 发生第二次 Heck 反 应: 氧化加成生成中间体 $\mathbf{I 4}$, 然后还原消除得目标产物 氧化芪三酚，同时钯催化剂得以循环.

\section{2 结论}

从 3,5-二甲氧基苯胺和间苯二酚出发, 分别合成中 间体 3,5-二羟基碘苯和 2,4-二羟基碘苯, 然后在钯催化 剂的作用下依次发生 Heck 反应、脱羧反应和 Heck 反应, 得到目标产物氧化芪三酚. 该方法具有合成步骤少、产 率高和操作简单等特点, 为合成氧化芪三酚提供了一个 新的方法.

\section{3 实验部分}

\section{1 仪器与试剂}

核磁共振谱采用德国 Brucker $400 \mathrm{MHz}$ ADVANCE 


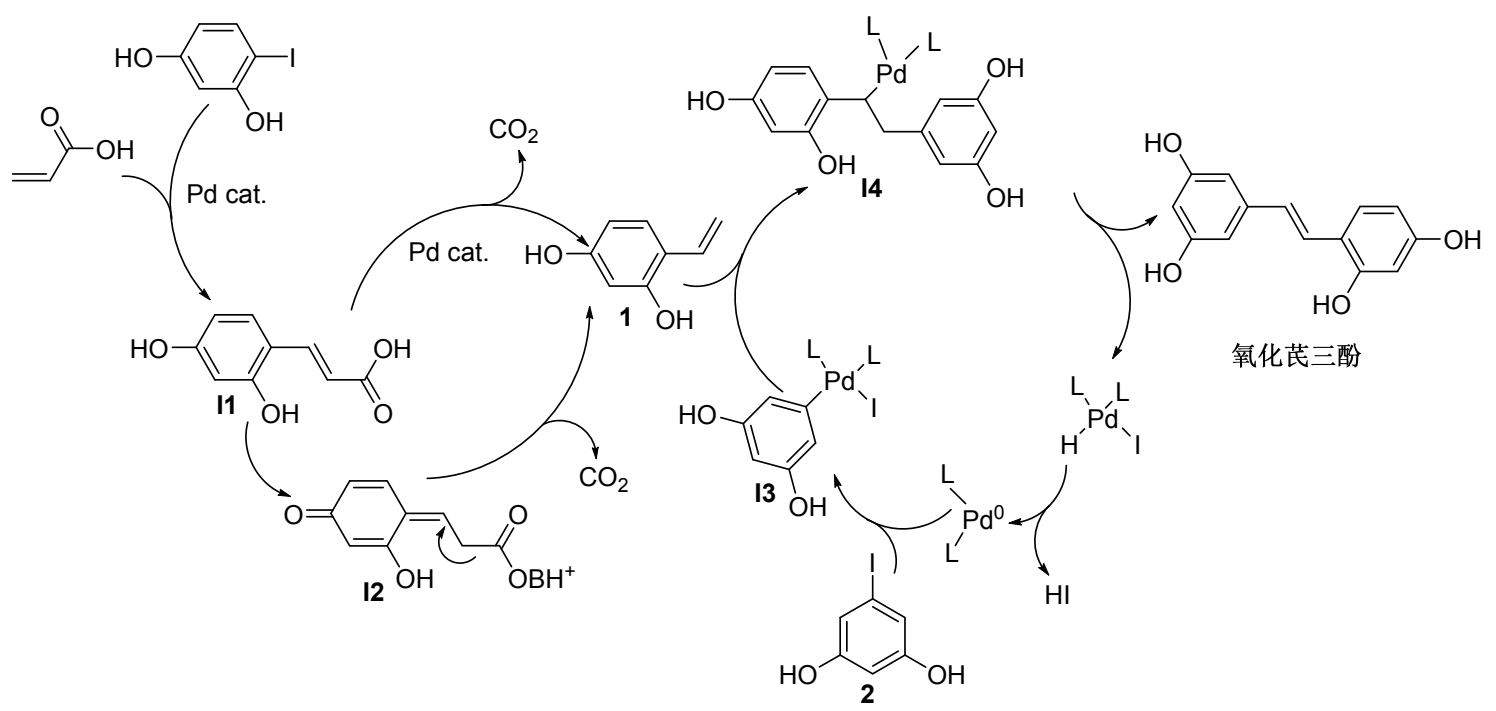

图 1 合成氧化芪三酚的可能机理

Figure 1 Proposed mechanism for the synthesis of oxyresveratrol

DMX 500 型核磁共振仪测定, TMS 为内标; ESI 质谱采 用德国 Brucker Esquire 600 型质谱仪测定; 熔点采用 X-4 数字显示显微熔点测定仪测定, 温度未加校正.

柱层析采用 200 300 目层析硅胶粉(青岛海洋化工 厂); 薄层色谱采用 GF254 硅胶板(青岛海洋化工厂); 四 (三苯基膦) 钯和芐基三苯基高氯酸鏻(BTPPC)自制; 其 它所用化学试剂均为市售分析纯, 使用前未进一步纯 化.

\section{2 实验方法}

\subsection{1 中间体的合成}

3,5-二羟基碘苯参考文献[15]合成, m.p. 94 96 ${ }^{\circ} \mathrm{C}$ (文献值 ${ }^{[15]}$ m.p. $95 \sim 96{ }^{\circ} \mathrm{C}$ ); ESI-MS, $m / z$ : $237.1[\mathrm{M}+$ $\mathrm{H}]^{+}$.

2,4-二羟基碘苯参考文献[16]合成, m.p. $66 \sim 68{ }^{\circ} \mathrm{C}$ (文献值 ${ }^{[16]}$ m.p. $67 \sim 70{ }^{\circ} \mathrm{C}$ ); ESI-MS $m / z$ : $237.2[\mathrm{M}+$ $\mathrm{H}]^{+}$.

\subsection{2 目标化合物的合成}

在配有磁力搅拌器和回流冷凝管的 $50 \mathrm{~mL}$ 圆底烧 瓶中加入 $0.014 \mathrm{~g}$ 氯化锂 $(0.332 \mathrm{mmol}) 、 0.192 \mathrm{~g}$ 四(三苯 基膦)钯 $(0.166 \mathrm{mmol}) 、 0.698 \mathrm{~g}$ 氢氧化钾 $(12.48 \mathrm{mmol})$ 、

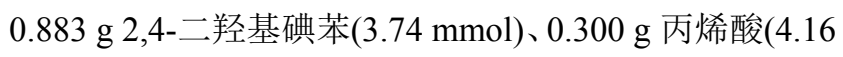
$\mathrm{mmol}$ )和 $3 \mathrm{~mL}$ 蒸馏水, 在 $85{ }^{\circ} \mathrm{C}$ 的油浴中加热搅拌反应 $3 \mathrm{~h}$. 将反应体系冷却至室温后, 再加入 $0.005 \mathrm{~g}$ 氯化锂 $(0.124 \mathrm{mmol}) 、 0.144 \mathrm{~g}$ 四(三苯基膦)钯 $(0.124 \mathrm{mmol})$ 、

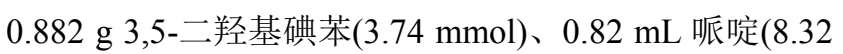
$\mathrm{mmol}$ )和 $12 \mathrm{~mL} \mathrm{~N}, \mathrm{~N}$-二甲基乙酰胺, 在 $140{ }^{\circ} \mathrm{C}$ 油浴中反 应 $14 \mathrm{~h}$. 反应完成后将体系冷却至室温, 过滤. 滤液中 加入稀盐酸使呈酸性 $(\mathrm{pH}=5)$. 倒入分液漏斗中, 用乙
酸乙酯萃取 $(20 \mathrm{~mL} \times 3)$. 合并有机相, 有机层用无水硫 酸钠干燥, 过滤, 旋除溶剂后得黄色油状液体, 柱层析 [洗脱剂: $V$ (乙酸乙酯) : $V$ (石油醚) $=1: 2$ ] 后, 得 $0.521 \mathrm{~g}$ 浅黄色固体, 产率 $57.1 \%$. m.p. 201 203 ${ }^{\circ} \mathrm{C}$ (文献值 ${ }^{[7]}$ m.p. 202 205 ${ }^{\circ} \mathrm{C}$; 文献值 ${ }^{[17]}$ m.p. $\left.199 \sim 200{ }^{\circ} \mathrm{C}\right) ;{ }^{1} \mathrm{H}$ NMR (DMSO- $\left.d_{6}, 400 \mathrm{MHz}\right) \delta: 6.06(\mathrm{t}, J=2.0 \mathrm{~Hz}, 1 \mathrm{H})$, $6.23(\mathrm{dd}, J=2.4,8.4 \mathrm{~Hz}, 1 \mathrm{H}), 6.31(\mathrm{~d}, J=2.0 \mathrm{~Hz}, 1 \mathrm{H})$, $6.33(\mathrm{~d}, J=1.6 \mathrm{~Hz}, 2 \mathrm{H}), 6.76(\mathrm{~d}, J=16.4 \mathrm{~Hz}, 1 \mathrm{H}), 7.14(\mathrm{~d}$, $J=16.4 \mathrm{~Hz}, 1 \mathrm{H}), 7.34$ (d, $J=8.8 \mathrm{~Hz}, 1 \mathrm{H}), 9.17$ (s, 2H), $9.41(\mathrm{~s}, 1 \mathrm{H}), 9.60(\mathrm{~s}, 1 \mathrm{H}) ;{ }^{13} \mathrm{C}$ NMR (DMSO- $d_{6}, 100 \mathrm{MHz}$ ) $\delta: 101.4,102.6,104.0,107.3,115.3,123.2,124.6,127.2$, 140.1, 156.6, 158.2, 158.6; ESI-MS $m / z: 245.3[\mathrm{M}+\mathrm{H}]^{+}$.

辅助材料(Supporting Information) 目标化合物氧化 芪三酚的 ${ }^{1} \mathrm{H}$ NMR、 ${ }^{13} \mathrm{C}$ NMR 和 ESI-MS 图谱. 这些材 料可以免费从本刊网站(http://sioc-journal.cn/)上下载.

\section{References}

[1] Li, H. T.; Cheng, K. W.; Cho, C. H.; He, Z. D.; Wang, M. F. J. Agric. Food Chem. 2007, 55, 2604.

[2] Park, J.; Park, J. H.; Suh, H. J.; Lee, I. C.; Koh, J.; Boo, Y. C. Arch. Dermatol. Res. 2014, 306, 475.

[3] Weber, J. T.; Lamont, M.; Chibrikova, L.; Fekkes, D.; Vlug, A. S.; Lorenz, P.; Kreutzmann, P.; Slemmer, J. E. Eur. J. Pharmacol, 2012, 680, 55.

[4] Suhee, S.; Hyojin, L.; Youngeup, J.; Young, M. H.; Sungjin, B.; Hae, Y. C.; Hongsuk, S. Bioorg. Med. Chem. Lett. 2007, 17, 461.

[5] Taksina, C.; Jurairatana, P.; Vimolmas, L.; Kittisak, L.; Mikiko, S.; Pornpen, P.; Masao, H.; Kimiyasu, S. Antiviral Res. 2008, 80, 62.

[6] Choi, S. Y.; Kim, S.; Hwang, J. S.; Lee, B. G.; Kim, H.; Kim, S. Y. Biochem. Pharmacol. 2004, 67, 707.

[7] Sun, H. Y.; Xiao, C. F.; Wen, W.; Chen, Y.; Lü, Z. L.; Zou, Y. Chin. 
J. Org. Chem. 2010, 30, 1574 (in Chinese).

(孙洪宜，肖春芬，魏文，陈显，吕泽良，邹永，有机化学，2010, 30, 1574.)

[8] Zheng, Q. Y.; Li, Z. W.; Xie, Z. Y.; Bao, Z. C.; Wang, J. N. CN 103172499, 2013 [Chem. Abstr. 2013, 159, 181645].

[9] Wu, X. F.; Anbarasan, P.; Neumann, H.; Beller, M. Angew. Chem., Int. Ed. 2010, 49, 9047.

[10] Oestreich, M. The Mizoroki-Heck Reaction, Wiley, Weinheim, 2009, Chapter 1.

[11] Fu, Z. J.; Li, Z. J.; Xiong, Q. H.; Cai, H. Chin. J. Org. Chem. 2015, 35, 984 (in Chinese).

(付拯江, 李兆杰, 熊起恒, 蔡琥, 有机化学, 2015, 35, 984.)

[12] Zhou, Q. Z.; Zhang, B.; Chen, D.; Chen, R. E.; Jiang, H. J. Chin. J.
Org. Chem. 2011, 31, 2181 (in Chinese).

(周其忠, 张斌, 陈丹, 陈仁尔, 蒋华江, 有机化学, 2011, 31, 2181.)

[13] Prakash, G. K. S.; Jog, P. V.; Krishnan, H. S.; Olah, G. A. J. Am. Chem. Soc. 2011, 133, 2140.

[14] Shard, A.; Sharma, N.; Bharti, R.; Dadhwal, S.; Kumar, R.; Sinha, A. K. Angew. Chem., Int. Ed. 2012, 51, 12250.

[15] Mao, W. W.; Wang, T. T.; Zeng, H. P.; Wang, Z. Y.; Chen, J. P.; Shen, J. G. Bioorg. Med. Chem. Lett. 2009, 19, 4570.

[16] Albadi, J.; Abedini, M.; Iravani, N. Chin. Chem. Lett. 2012, 23, 261.

[17] Tuba, A.; Ahmet, C.; Cavit, K.; Neslihan, B.; Yasin, B.; Yavuz, T. Chem. Biodiversity 2014, 8, 1192.

(Qin, X.) 